\title{
Safety and Efficacy of Physician-Supervised Self-Managed C1 Inhibitor Replacement Therapy
}

\author{
Louanne M. Tourangeau $^{a}$ Anthony J. Castaldo ${ }^{b}$ Donna K. Davis ${ }^{b}$ \\ James Koziolc Sandra C. Christiansen ${ }^{\mathrm{a}}{ }^{\mathrm{d}}$ Bruce L. Zuraw ${ }^{\mathrm{a}}$ e

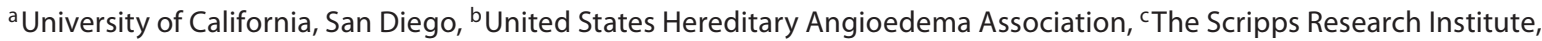

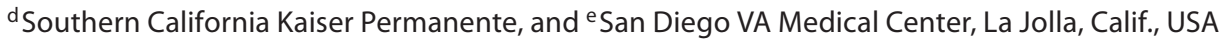

\section{Key Words}

C1 inhibitor $\cdot$ Hereditary angioedema $\cdot$ Home infusion $\cdot$

C1 inhibitor deficiency

\begin{abstract}
Background: $\mathrm{C} 1$ inhibitor $(\mathrm{C} 1 \mathrm{NH})$ has recently been approved in the USA for the treatment of acute attacks in hereditary angioedema (HAE) patients. The literature suggests that treatment with $\mathrm{C} 1 \mathrm{INH}$ is most effective when administered early in an attack. Home infusion of C1INH allows for the earliest possible intervention since patients can initiate therapy at the first sign of symptoms. Methods: We performed an observational, prospective study on 39 subjects with HAE utilizing two groups of patients: one receiving ondemand $\mathrm{C} 1 \mathrm{INH}$ replacement therapy in a medical facility and the other self-managing on-demand $\mathrm{C} 1 \mathrm{INH}$ replacement therapy in the home setting under the supervision of a treating physician. All subjects completed online questionnaires weekly for 8 weeks. Results: There were statistically significant decreases in attack duration $(p<0.0001)$, pain medication use $(p<0.0001)$ and graded attack severity $(p<0.005)$ in the subjects who received $\mathrm{C} 1 \mathrm{INH}$ in the home setting versus the clinic-based group. Attack frequency was similar between the groups. The home group experienced more
\end{abstract}

frequent injection-related side effects; however, the clinic group noted more severe adverse events from $\mathrm{C} 1 \mathrm{INH}$. Conclusion: Physician-supervised self-managed C1INH replacement therapy is a safe and effective treatment for patients with HAE with potential benefits in diminishing attack duration and attack severity.

Copyright $\odot 2011$ S. Karger AG, Basel

\section{Introduction}

Hereditary angioedema (HAE) is a rare, autosomal dominant disease that is clinically characterized by recurrent episodes of subcutaneous or submucosal edema. Common sites for swelling include the extremities, bowels, genitalia, face, tongue, or larynx. Angioedema attacks typically begin in the first decade, worsen with puberty and persist throughout life. Disease severity is variable and untreated HAE patients experience attacks every $1-2$ weeks on average, with the frequency ranging from almost never to as often as every 3 days $[1,2]$. Angioedema attacks often follow a predictable course with swelling that increases slowly but continuously over the first $24 \mathrm{~h}$ and then gradually abates over the following 48-72 h. HAE attacks involving the oropharynx or the abdomen

\section{KARGER \\ Fax +41613061234 E-Mail karger@karger.ch}

(C) 2011 S. Karger AG, Basel www.karger.com www.karger.com/iaa
Correspondence to: Dr. Bruce L. Zuraw

University of California, San Diego

9500 Gilman Dr.

La Jolla, CA 92093-0732 (USA)

Tel. +1 858822 6597, E-Mail bzuraw@ucsd.edu 
are associated with significant risk of morbidity and mortality $[3,4]$.

Genetically, HAE is characterized by mutations in the SERPING1 gene [5] and can be divided into 2 major types. Type I HAE is defined by decreased antigenic and functional $\mathrm{C} 1$ inhibitor (C1INH) levels, whereas type II HAE has normal C1INH antigenic levels but decreased C1INH functional levels. Clinically, type I and II HAE present in a similar fashion. More recently, a third type of familial angioedema has been described which is associated with normal $\mathrm{C} 1 \mathrm{INH}$ antigenic and functional levels and sometimes associated with a defect in factor XII (Hageman factor) [6, 7] and/or estrogen dependency.

Due to the morbidity and mortality caused by recurrent swelling attacks in HAE, medical intervention is both necessary and life saving for many patients. Treatment includes management of acute attacks, short-term prophylactic therapy to prevent future attacks, and longterm prophylaxis. Recently, C1 inhibitor replacement therapy has become available in the US and has been shown to be highly effective for both acute and prophylactic management of HAE attacks [8-11]. Administration of C1INH rapidly halts attack progression [12-15], with improvement typically noted within 30-60 min.

Theoretical considerations as well as clinical observations suggest that $\mathrm{C} 1 \mathrm{INH}$ replacement is most effective when administered early in an attack [16-23]. While patients typically receive acute attack treatment at a clinic or hospital, several studies suggest that home infusion of $\mathrm{C} 1 \mathrm{INH}$ is safe and provides a superior outcome compared to treatment at a medical facility [17-20, 24]. As a result, a recent HAE international home therapy consensus recommends that every patient with physician-diagnosed HAE be considered for physician-supervised, selfmanaged home $\mathrm{CIINH}$ replacement therapy and that therapy should be started as soon as the patient notices symptoms of an HAE attack [25]. We hypothesized that physician-supervised self-managed $\mathrm{C} 1 \mathrm{INH}$ home replacement therapy would result in better control of $\mathrm{HAE}$ attacks compared to clinic treatment with $\mathrm{C} 1 \mathrm{INH}$. The aim of this study was to investigate the safety and efficacy of self-managed home $\mathrm{ClINH}$ replacement compared to $\mathrm{C} 1 \mathrm{INH}$ given in the clinical setting.

\section{Methods}

\section{Study Design}

The United States HAE Association conducted an investigator-designed survey of subjects with physician-diagnosed HAE who were receiving on-demand C1INH either at home or in a medical facility from December 2007 to March 2008. C1INH was provided in the form of a human plasma-derived concentrate from Sanguin (Cetor) or Lev Pharmaceuticals (Cinryze). C1INH was administered by intravenous injection by either trained medical personnel at a medical facility (clinic group) or by the patient or family member away from a medical facility (home group). The C1INH dose was 1,000 units, irrespective of subject weight. Most subjects in both groups used 1 dose per attack; however, subjects could use a second 1,000-unit dose if the first dose was not effective. The median dose per attack was 1,000 units for all patients included in the study; however, the clinic group used a small but significantly higher mean dose of C1INH (1,014 \pm 120 units) than the home group (916 \pm 187 units; $\mathrm{p}<0.0001)$. The Western Institutional Review Board approved this study.

After subjects signed informed consent, they were given a password for online access to a computerized questionnaire consisting of three parts: (1) a health survey to determine past medical history, (2) an interval history to obtain information regarding attack frequency, attack severity, amount of medication used for the previous week, and (3) a Medical Outcomes Study 36-Item Short Form Health Survey (SF-36) at the beginning and end of the study. Thirty-nine subjects receiving on-demand C1INH in the home or clinic were enrolled and divided into two groups. The first group consisted of 18 subjects receiving on-demand intravenous C1INH infusions at a clinic or a medical facility, and the second group consisted of 21 subjects who self-administered ondemand intravenous $\mathrm{C} 1 \mathrm{INH}$ as replacement therapy under the supervision of their physician. Both groups were treated with C1INH concentrate on demand for acute attacks of angioedema. Subjects filled out the online form weekly for 8 weeks.

Subjects in the self-administration group received training in the home or in a medical facility. A registered nurse provided training in the majority of cases; however, 3 of the patients felt comfortable with intravenous infusion based on prior knowledge/ training, a career in health care, or a family member in health care. Two subjects received training directly from a physician. Although the subjects in the home group self-infused $\mathrm{C} 1 \mathrm{INH}$ away from a medical facility, all subjects reported that their physician supervised the process, including initiating the infusion training, periodic monitoring of the dose and use of C1INH, as well as routine follow-up.

Each study subject underwent patient-specific training based on his or her level of comfort and knowledge. The time for each patient to practice intravenous initiation ranged from $0 \mathrm{~min}$ to $3 \mathrm{~h}$. Each patient remained in training until they felt comfortable initiating their own intravenous infusion. Depending on each patient's level of comfort, patients were given the opportunity to practice on oranges, family members, health care professionals and/or themselves. Following successful initiation of an intravenous infusion, subjects were allowed to practice until they felt completely comfortable with the procedure. The entire training period from beginning to proficiency ranged from $10 \mathrm{~min}$ to $10 \mathrm{~h}$. After training, all patients reported that they felt comfortable with self-infusion in their home.

The primary endpoint was the duration of attacks treated during the study. Secondary endpoints included the number of attacks over the 8-week period, the severity of attacks, use of narcotic pain medications, and the safety and tolerability of the therapy. 
Table 1. Subject demographics and retrospective disease severity

\begin{tabular}{|c|c|c|c|c|c|}
\hline \multirow[t]{2}{*}{ Parameter } & \multicolumn{2}{|c|}{ Clinic group $(\mathrm{n}=18)$} & \multicolumn{2}{|c|}{ Home group $(n=21)$} & \multirow[t]{2}{*}{ p value $^{1}$} \\
\hline & mean $\pm \mathrm{SD}$ & median (IQR) & mean $\pm \mathrm{SD}$ & median (IQR) & \\
\hline Age at start of study, years & $39.78 \pm 10.96$ & $40(34.25-43.75)$ & $38.30 \pm 14.61$ & $34(27-49)$ & 0.573 \\
\hline Gender (female:male) & $16: 2$ & & $15: 6$ & & 0.191 \\
\hline Age at HAE symptom onset, years & $9.15 \pm 6.29$ & $8(4-16)$ & $6.60 \pm 4.97$ & $5(3-11)$ & 0.310 \\
\hline Age at HAE diagnosis, years & $16.92 \pm 8.85$ & $19(12-24)$ & $16.75 \pm 10.71$ & $15(10-21)$ & 0.480 \\
\hline Known family history of HAE (yes:no) & $17: 1$ & & $13: 8$ & & 0.019 \\
\hline Lifetime ED visits & $133.7 \pm 313.0$ & $45(4-115)$ & $101.7 \pm 166.1$ & $50(10-100)$ & 0.81 \\
\hline Missed work/school days per year & $39.0 \pm 44.0$ & $15(10-43.7)$ & $128.5 \pm 31.2$ & $50(20-144)$ & 0.04 \\
\hline Lifetime hospitalizations & $90.9 \pm 237.8$ & $14(2-37.5)$ & $57.7 \pm 136.2$ & $10(3-50)$ & 0.97 \\
\hline Lifetime surgeries & $0.67 \pm 1.28$ & $0(0-1)$ & $0.20 \pm 0.52$ & $0(0-0)$ & 0.15 \\
\hline Lifetime intubations & $0.5 \pm 1.34$ & $0(0-0)$ & $0.8 \pm 2.69$ & $0(0-0)$ & 0.90 \\
\hline Average attacks per year & $78.7 \pm 47.9$ & $81(38.5-111.25)$ & $94.0 \pm 54.6$ & $99(60-122)$ & 0.28 \\
\hline Average annual extremity attacks & $28.1 \pm 17.9$ & $24(12-46)$ & $32.1 \pm 20.0$ & $39(12-51)$ & 0.45 \\
\hline Average annual facial attacks & $6.5 \pm 11.8$ & $2(1-6)$ & $7.15 \pm 11.5$ & $4(2-6)$ & 0.71 \\
\hline Average annual throat attacks & $6.0 \pm 12.5$ & $1.5(1-5)$ & $6.0 \pm 11.9$ & $2.5(1-4)$ & 1.00 \\
\hline Average annual genitourinary attacks & $11.1 \pm 13.2$ & $7(2-15)$ & $12.2 \pm 12.0$ & $7(4-20)$ & 0.37 \\
\hline Average annual abdominal attacks & $27.1 \pm 17.1$ & $22(12-40)$ & $36.7 \pm 17.0$ & $47.5(24-51)$ & 0.06 \\
\hline
\end{tabular}

$\mathrm{ED}=$ Emergency department.

${ }^{1}$ Mann-Whitney rank-sum test for continuous variables; Fisher's exact test for discrete variables.

Statistical Analysis

Results are reported both as the mean $\pm \mathrm{SD}$ as well as the median with interquartile range (IQR). Because most of the endpoints had non-Gaussian distributions, we utilized non-parametric procedures, primarily the Wilcoxon-Mann-Whitney test, for comparing the clinic and home groups. Attacks were taken as individual events. A p value $\leq 0.05$ was considered statistically significant.

\section{Results}

\section{Study Population}

The clinic group consisted of 18 subjects and the home group included 21 subjects. All study participants had physician-diagnosed HAE. Baseline characteristics and historical measures of disease severity for the two groups were compared based on their initial health survey questionnaire (table 1). No differences were seen in age at entry, gender, age at symptom onset, age at diagnosis, number of lifetime emergency department visits, lifetime hospitalizations, total intubations, total number of attacks/ year, or number of attacks/year involving the extremities, face, throat, genitourinary tract, or abdomen between the two groups. Both groups had relatively severe HAE, based on the average number of attacks per year: a median of 81 total attacks/year (IQR 38.5-111.25) in the clinic group and 99 attacks/year (IQR 60-122) in the home group. The home group did report significantly more missed days of work or school per year (50, IQR 20-144) than did the clinic group (15, IQR 10-43.7; $\mathrm{p}=0.04)$. The home group used C1INH a total of 167 times (0-17 injections per patient over 8 weeks, median 7, IQR 2-13), whereas the clinic group used C1INH a total of 69 times $(0-18$ injections per patient over 8 weeks, median 2, IQR 0-6.5).

\section{Impact of Home C1INH Replacement Therapy on \\ Number and Severity of Acute Attacks}

As expected, the weekly attack frequency was not significantly different between the two groups (clinic group, median 1, IQR 0-2; home group, median 1, IQR 0-2; p = 0.82 ) (table 2). Subjects also graded the overall severity of their attacks on a scale of 1-10. The home treatment group reported a statistically significant lower attack severity (median 4 , IQR $3-7$ ) than the clinic treatment group (median 5, IQR 4-8; p < 0.005) (fig. 1; table 2).

\section{Efficacy of Home versus Clinic C1INH Replacement \\ Therapy for Acute Attacks}

Subject-reported efficacy of C1INH for acute attacks was similar in the two cohorts. $87.2 \%$ of clinic subjects who received clinic C1INH replacement therapy and $86.8 \%$ of home subjects who received $\mathrm{C} 1 \mathrm{INH}$ as home re- 
Table 2. Interval efficacy and safety/tolerability results

\begin{tabular}{|c|c|c|c|c|c|}
\hline \multirow[t]{2}{*}{ Parameter } & \multicolumn{2}{|c|}{ Clinic group $(\mathrm{n}=18)$} & \multicolumn{2}{|c|}{ Home group $(\mathrm{n}=21)$} & \multirow[t]{2}{*}{$\mathrm{p}$ value ${ }^{1}$} \\
\hline & mean $\pm \mathrm{SD}$ & median (IQR) & mean $\pm \mathrm{SD}$ & median (IQR) & \\
\hline \multicolumn{6}{|l|}{ Attack frequency/severity } \\
\hline Average attacks/week & $0.83 \pm 0.98$ & $1(0-2)$ & $1.02 \pm 0.96$ & $1(0-2)$ & 0.82 \\
\hline Attack severity (scale of $1-10$ ) & $5.67 \pm 2.69$ & $5(4-8)$ & $4.75 \pm 2.46$ & $4(3-7)$ & $<0.005$ \\
\hline \multicolumn{6}{|l|}{ Efficacy } \\
\hline Attack duration, h & $34.2 \pm 27.5$ & $30.5(12-48)$ & $8.0 \pm 13.5$ & $3.0(1.5-6.0)$ & $<0.0001$ \\
\hline Pain medications per week & $1.75 \pm 3.13$ & $0(0-3)$ & $0.38 \pm 0.98$ & $0(0-0)$ & $<0.0001$ \\
\hline \multicolumn{6}{|l|}{ Safety/tolerability results } \\
\hline Bleeding from injection site, $\%$ & $0.58 \pm 7.6$ & $0(0-0)$ & $7.81 \pm 26.9$ & $0(0-0)$ & $<0.001$ \\
\hline Pain/inflammation at injection site, $\%$ & $1.73 \pm 13.1$ & $0(0-0)$ & $7.21 \pm 25.9$ & $0(0-0)$ & 0.009 \\
\hline Infection at injection site, $\%$ & $0 \pm 0$ & $0(0-0)$ & $0.30 \pm 5.5$ & $0(0-0)$ & 0.47 \\
\hline Difficulty with injection (scale of $0-3$ ) & $0.03 \pm 0.17$ & $0(0-0)$ & $0.31 \pm 0.60$ & $0(0-0)$ & $<0.0001$ \\
\hline Severity of C1INH side effects (scale of $0-3$ ) & $0.22 \pm 0.71$ & $0(0-0)$ & $0.01 \pm 0.16$ & $0(0-0)$ & $<0.0001$ \\
\hline
\end{tabular}

${ }^{1}$ Mann-Whitney rank-sum test.

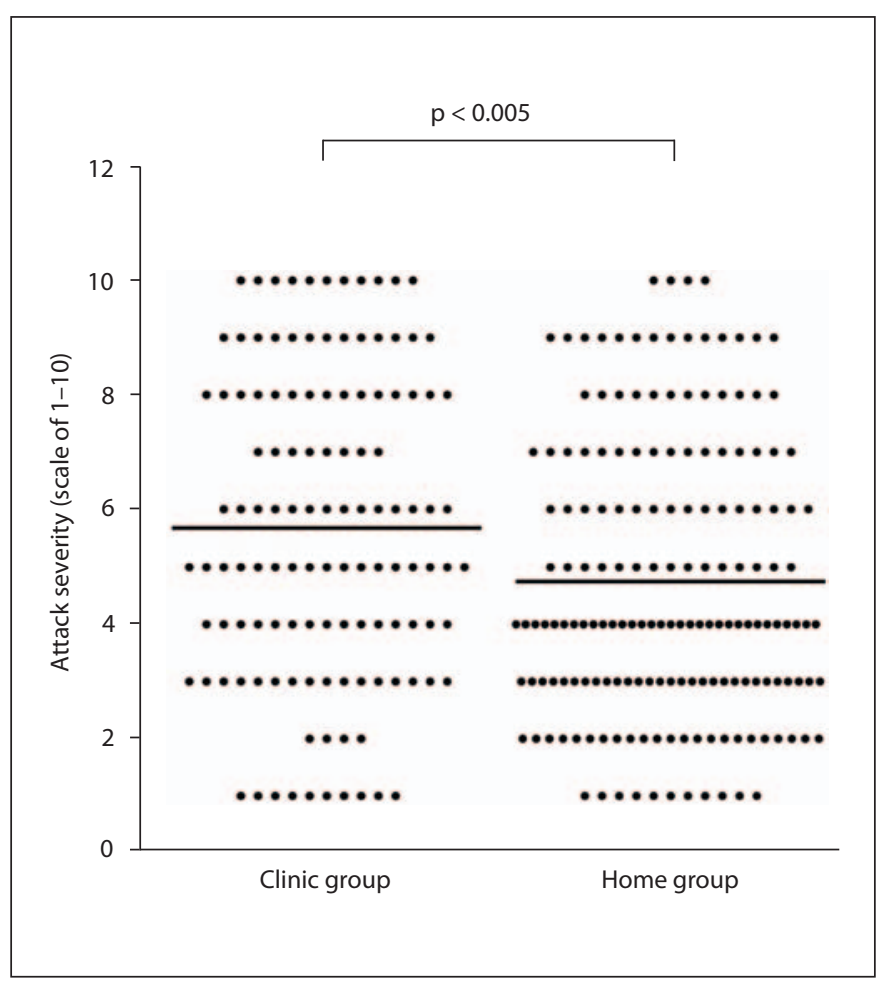

Fig. 1. Severity of angioedema attacks in the clinic and home treatment groups $(\mathrm{p}<0.005)$. Each attack is shown as a single black filled circle. Subjects graded each attack from 1 to 10 (1 being very mild and 10 being very severe). The mean severity for each group is shown as a straight line.

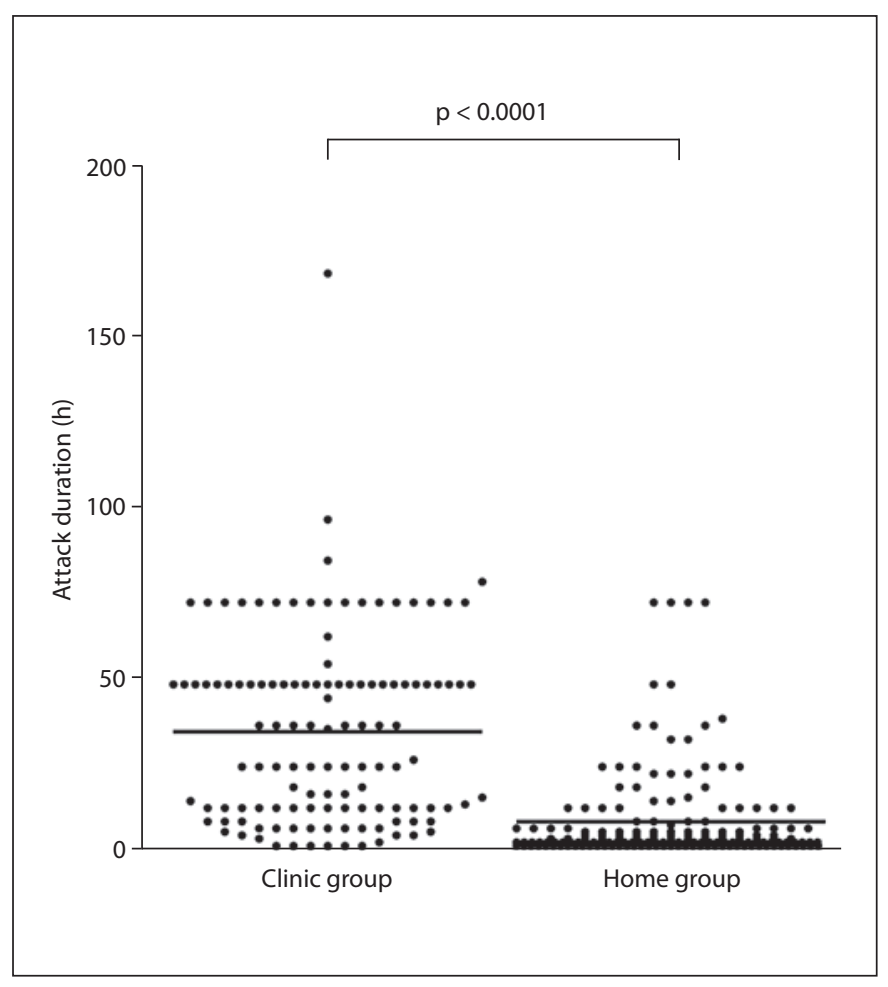

Fig. 2. Duration of angioedema attacks in the clinic and home treatment groups $(\mathrm{p}<0.0001)$. Each attack is shown as a single black filled circle. The mean duration for each group is shown as a straight line. 
Table 3. Impact on life activities and SF-36 data

\begin{tabular}{|c|c|c|c|c|c|}
\hline \multirow[t]{2}{*}{ HAE impact or SF-36 score } & \multicolumn{2}{|c|}{ Clinic group $(\mathrm{n}=18)$} & \multicolumn{2}{|c|}{ Home group $(\mathrm{n}=21)$} & \multirow[t]{2}{*}{ p value ${ }^{1}$} \\
\hline & mean $\pm \mathrm{SD}$ & median (IQR) & mean $\pm \mathrm{SD}$ & median (IQR) & \\
\hline Negative impact on school/job ${ }^{2}$ & $0.48 \pm 0.92$ & $0(0-1)$ & $0.42 \pm 0.79$ & $0(0-1)$ & 0.886 \\
\hline Negative impact on family life ${ }^{2}$ & $1.09 \pm 1.09$ & $1(0-2)$ & $0.53 \pm 0.83$ & $0(0-1)$ & $<0.0001$ \\
\hline Negative impact on social life ${ }^{2}$ & $1.02 \pm 1.12$ & $1(0-2)$ & $0.51 \pm 0.91$ & $0(0-1)$ & $<0.0001$ \\
\hline Days missed school/job per week & $0.56 \pm 3.46$ & $0(0-0)$ & $0.18 \pm 0.53$ & $0(0-0)$ & 0.687 \\
\hline \multicolumn{6}{|l|}{ Days family/friends missed school/job } \\
\hline per week due to subject's HAE & $0.25 \pm 0.73$ & $0(0-0)$ & $0.10 \pm 0.40$ & $0(0-0)$ & 0.045 \\
\hline Nights of disrupted sleep per week & $1.61 \pm 4.39$ & $1(0-2)$ & $0.50 \pm 1.07$ & $0(0-1)$ & $<0.0001$ \\
\hline SF-36 initial physical aggregate score & $36.52 \pm 3.38$ & $37.10(34.12-38.75)$ & $35.91 \pm 3.33$ & $36.29(33.83-38,17)$ & 0.749 \\
\hline SF-36 final physical aggregate score & $37.00 \pm 2.38$ & $36.69(35.14-38.01)$ & $38.77 \pm 2.69$ & $38.37(37.34-41.11)$ & 0.040 \\
\hline Change in physical aggregate score & $0.49 \pm 2.81$ & $0.49(-1.14$ to 2.50$)$ & $2.86 \pm 3.87$ & $2.23(0.78-5.56)$ & 0.043 \\
\hline SF-36 initial mental aggregate score & $30.92 \pm 6.85$ & $31.06(25.59-37.38)$ & $30.52 \pm 6.39$ & $30.13(24.90-36.33)$ & 0.813 \\
\hline SF-36 final mental aggregate score & $32.30 \pm 7.97$ & $35.96(25.59-38.09)$ & $34.66 \pm 6.31$ & $36.41(30.53-39.45)$ & 0.321 \\
\hline Change in mental aggregate score & $1.38 \pm 5.74$ & $1.01(-0.72$ to 2.82$)$ & $4.14 \pm 7.69$ & $3.08(-0.53$ to 10.49$)$ & 0.257 \\
\hline
\end{tabular}

${ }^{1}$ Mann-Whitney rank-sum test. ${ }^{2}$ Graded on a scale of $0-3$.

placement therapy reported that therapy was effective for controlling attacks. Attack duration, the primary endpoint, was dramatically shorter in the home group $(3.0 \mathrm{~h}$, IQR $1.5-6.0)$ compared to the clinic group ( $30.5 \mathrm{~h}$, IQR 12-48; $\mathrm{p}<0.0001$ ) (fig. 2; table 2). Of note, despite the similar frequency of attacks, the home treatment group used CIINH replacement significantly more often than the clinic infusion group ( $\mathrm{p}<0.0001)$. Repeating the analysis of duration only for attacks that were treated with C1INH did not alter the outcome, with a significantly shorter median duration of attack still observed in the home group (home group, median $2.0 \mathrm{~h}$, IQR 1.25-6; clinic group, median $13.0 \mathrm{~h}$, IQR 6-48; p < 0.0001). Pain medication use was assessed as a surrogate marker for efficacy. Consistent with the primary efficacy results, the home group reported a significant decrease in prescription pain medication use compared to the clinic group $(\mathrm{p}<0.0001)$ (fig. 3; table 2).

\section{Impact of C1INH Replacement Therapy on Life \\ Activities}

The effect of home versus clinic C1INH administration on the negative impact of HAE on life activities was assessed as a secondary efficacy outcome. On a weekly basis, subjects answered questions regarding the impact of their HAE on various life activities using a scale from 0 (no interference) to 3 (significant negative impact) (table 3). The home group reported statistically less interference with family life $(\mathrm{p}<0.0001)$, social life $(\mathrm{p}<0.0001)$, nights of disrupted sleep ( $\mathrm{p}<0.0001)$, and days that family or friends had to miss school or work due to the subject's HAE $(p=0.045)$ compared to the clinic group (table 3).

\section{Tolerability and Adverse Effects of C1INH Replacement Therapy}

A greater number of adverse effects were reported with the injections in the home CIINH replacement group (table 2). There was a statistically significant increase in the percentage of injections leading to bleeding events $(p<0.001)$, pain $(p=0.009)$ and difficulty with the injection $(\mathrm{p}<0.0001)$ in the home group. No difference was observed in the rate of injection site infections. Almost all of these side effects were minor, and no serious adverse events were reported in either group. Interestingly, the clinic group reported a significantly greater overall severity of side effects (graded on a scale of $0-3$, from none to severe) than what was reported by the home group ( $<<0.0001)$.

\section{Quality of Life Comparison between Study Groups \\ Based on SF-36 Data}

The impact of C1INH replacement therapy on quality of life is shown in table 3. At study entry, the aggregated physical score was similar in both groups (clinic group, median 37.10, IQR 34.12-38.75; home group, median 36.29, IQR 33.83-38.17; $\mathrm{p}=0.749)$; however, the home group showed a significantly greater improvement in the 


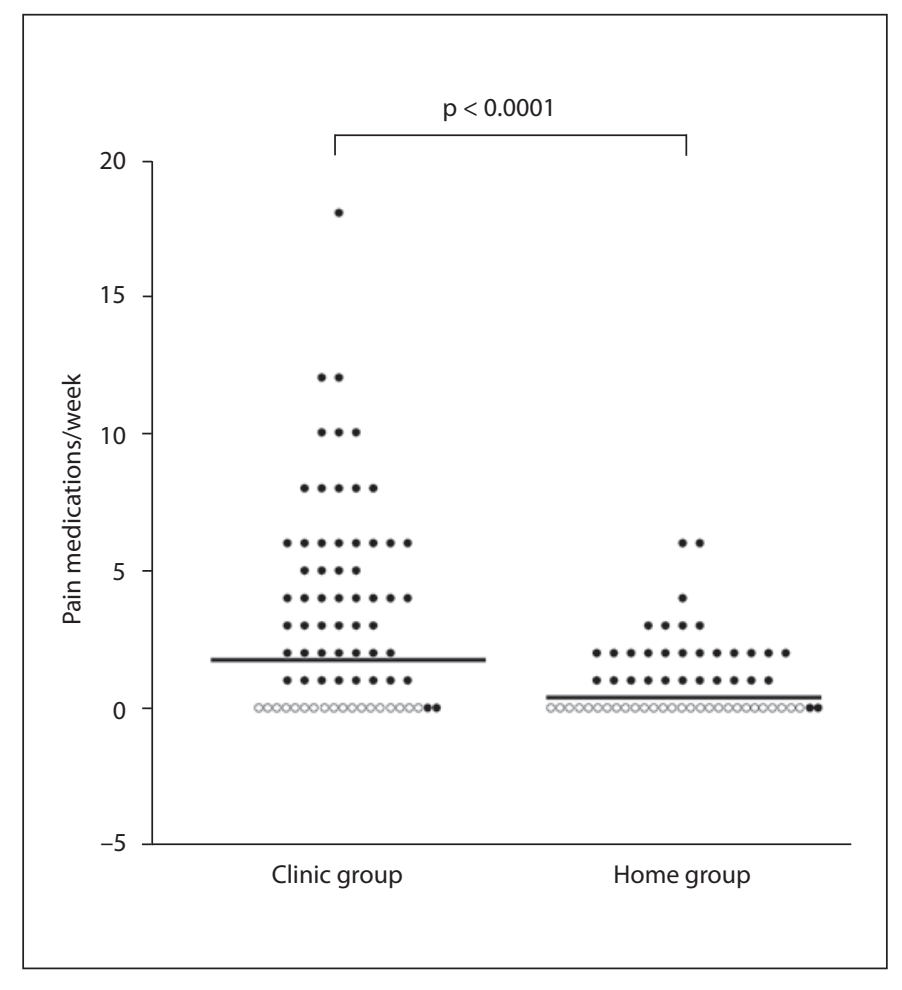

Fig. 3. Number of prescription pain medications used per week in the clinic and home treatment groups $(\mathrm{p}<0.0001)$. Each week is shown as a single black filled circle. Open circles represent 5 separate weeks, each requiring no pain medications. The mean weekly pain medication use for each group is shown as a straight line.

aggregated physical score over the course of the study (change in the clinic group, median 0.49 , IQR -1.14 to 2.50; change in the home group, median 2.23, IQR $0.78-$ 5.56; $\mathrm{p}=0.043)$. The aggregated mental score was also similar in both groups at entry (clinic group, median 31.06, IQR 25.59-37.38; home group, median 30.13, IQR $24.90-36.33 ; \mathrm{p}=0.813$ ), and improved more in the home group over the course of the study but failed to reach significance (change in the clinic group, median 1.01, IQR -0.72 to 2.82 ; change in the home group, median 3.08 , IQR -0.53 to 10.49 ).

\section{Discussion}

This study compared the efficacy, safety and tolerability of self-administered, physician-supervised on-demand C1INH replacement therapy in the home setting to on-demand C1INH replacement therapy in a medical facility. No difference was found in the ability of C1INH replacement therapy to reduce angioedema symptoms between the two groups; however, the home treatment group demonstrated highly significant reductions in total duration of angioedema attacks, maximal attack severity and requirement for pain medication use. There were no serious adverse effects in either group, and no subject stopped C1INH replacement therapy because of tolerability issues. The home group experienced more injection-related side effects than the clinic group, but had less severe overall side effects from the C1INH administration. Overall, home injection with $\mathrm{C} 1 \mathrm{INH}$ proved to be more efficacious and just as safe and tolerable as clinic administration for the treatment of acute attacks of angioedema in severely affected HAE patients.

The improved efficacy of physician-supervised selfmanaged C1INH home replacement therapy is likely a consequence of a decreased lag time between the first sign of an attack and the prompt initiation of therapy. By correcting the enhanced vascular permeability earlier in an attack, home treatment likely diminishes the amount of fluid moving from the vasculature into the interstitial space, decreases maximal severity of swelling, and consequently decreases the time to resolution. The significant reduction in median duration of attacks in the home group ( $\mathrm{p}<0.0001$ ) was clinically meaningful as shown by significant improvement in the need for prescription pain medications, decreased interference with life activities, and increased improvement in quality of life measures.

Drawing from the experience of hemophilia patients who have been administering clotting factors for prophylactic or emergent therapy since the 1980s, there has been a gathering consensus that home infusion is a superior means of treatment. Hemophilia patients and their physicians recognized some time ago that it was not practical for patients to visit an infusion center multiple times each week [26]. As a consequence, home therapy programs were established and shown to be feasible, beneficial to the patient and cost-effective [20]. Accordingly, home infusion is currently regarded as standard of care for hemophilia patients, extending even to the pediatric population where children can be started on home therapy before the age of two.

An emerging international consensus recommends that all HAE attacks be treated as soon as possible in order to limit the morbidity and mortality that may be associated with attacks. This study confirms that physician-supervised, self-administered home C1INH therapy is a safe and effective means to implement this strategy. Importantly, subjects receiving on-demand $\mathrm{C} 1 \mathrm{INH}$ in 
the clinic were also significantly less likely to seek treatment of acute attacks, an outcome that further highlights the advantage of self-administration.

Based on the improved outcomes we observed from self-administration of C1INH, we suggest that other effective treatments of acute HAE attacks may similarly benefit from early self-administration. Additional options for physician-supervised, self-administered HAE therapies may eventually include icatibant, ecallantide and recombinant human C1INH. Icatibant, a bradykinin B2 receptor antagonist administered by subcutaneous injection, was approved for treatment of acute attacks in Europe in 2008 and in the US in 2011. Ecallantide, a potent inhibitor of plasma kallikrein administered by subcutaneous injection, was approved for use in the USA for acute attacks in 2009; however, a black box warning for possible anaphylactic reactions has precluded its self-administration [27]. Rhucin, a recombinant human C1INH, recently completed two successful phase 3 studies and is under review by the Food and Drug Administration. Rhucin (Ruconest) was recently approved by the European Medicines Agency for Europe.

Self-administered C1INH replacement therapy also provides HAE patients with an improved sense of control over an extremely burdensome disease. A literature review performed by Longhurst et al. [20] revealed that patients who received self-administered C1INH either for prophylaxis or on demand experienced improved quality of life and decreased frequency, duration and severity of attacks.

In addition, our findings are consistent with a previous retrospective report by Levi et al. [19] that examined outcomes in 31 patients using on-demand C1INH treatment and 12 patients using C1INH prophylactic therapy compared to historical controls. In this study, self-treated subjects receiving on-demand $\mathrm{C} 1 \mathrm{INH}$ experienced significant improvement in both time to beginning of relief and time to resolution. Interestingly, the mean attack duration was $5.9 \pm 2.2 \mathrm{~h}$ in self-treated subjects compared to $13.8 \pm 2.9 \mathrm{~h}$ in conventionally treated subjects, which is similar to the effect we observed. Kreuz et al. [17] conducted a study of 22 patients with severe HAE that investigated the safety, efficacy and quality of life associated with self-administration of C1INH concentrate over danazol prophylaxis. They found a reduced incidence of attacks and superior efficacy for all quality of life variables investigated.

Several limitations of our study need to be addressed. We studied 39 severely affected HAE subjects. Although this is a relatively small sample size, it should be noted that HAE is a rare disease, and to our knowledge, this is the largest prospective study with statistically significant results. Nonetheless, the combination of a small sample size and high disease severity may limit the ability to generalize our study findings to the HAE population as a whole. Furthermore, our study was performed using an observational prospective design. It was not feasible to randomize subjects because certain subjects were not comfortable with self-injection, and other subjects did not live in close enough proximity to a medical facility to ensure safe travel to the facility for treatment of acute attacks. Additionally, the use of patient-reported outcomes as a clinical endpoint has been widely debated; however, the World Health Organization has taken the stance that patient involvement in their health care is not only desirable but a social, economic and technical necessity [28, 29]. Lastly, any voluntary patient survey may involve reporting and recall bias that could affect the results. To limit this problem, we asked patients to fill out their survey weekly in order to decrease recall errors.

In conclusion, our findings provide additional impetus to alter the standard of care for HAE by making physician-supervised self-administered $\mathrm{C} 1 \mathrm{INH}$ replacement therapy a mainstay of on-demand treatment. Our data, together with results from previous studies [19, 20], reveal that self-administration of C1INH for on-demand treatment of acute attacks of angioedema in HAE is safe and results in decreased attack duration, improved quality of life and better patient control over their disease state. We propose that physician-supervised self-managed on-demand C1INH replacement therapy is more effective and equally safe as clinic-based care. Consequently, we recommend that all HAE patients be provided with the option of C1INH self-replacement.

\section{Acknowledgements}

We would like to thank the HAE patients who participated in this study. Louanne M. Tourangeau, MD, is supported in her fellowship training by a NIH T32 AI 07469 grant. 


\section{References}

$\checkmark 1$ Agostoni A, Cicardi M: Hereditary and acquired C1INH deficiency: biological and clinical characteristics in 235 patients. Medicine (Baltimore) 1992;71:206-215.

-2 Bork K, Meng G, Staubach P, Hardt J: Hereditary angioedema: new findings concerning symptoms, affected organs, and course. Am J Med 2006;119:267-274.

>3 Nzeako UC, Frigas E, Tremaine WJ: Hereditary angioedema: a broad review for clinicians. Arch Intern Med 2001;161:2417-2429.

4 Frank MM, Gelfand JA, Atkinson JP: Hereditary angioedema: the clinical syndrome and its management. Ann Intern Med 1976;84: 580-593.

5 Davis AE 3rd: C1INH and hereditary angioneurotic edema. Annu Rev Immunol 1988;6: 595-628.

6 Binkley KE, Davis A 3rd: Clinical, biochemical, and genetic characterization of a novel estrogen-dependent inherited form of angioedema. J Allergy Clin Immunol 2000;106: 546-550.

7 Bork K, Barnstedt SE, Koch P, Traupe H: Hereditary angioedema with normal $\mathrm{C} 1 \mathrm{INH}$ activity in women. Lancet 2000;356:213-217.

-8 Craig TJ, Levy RJ, Wasserman RL, et al: Efficacy of human $\mathrm{C} 1$ esterase inhibitor concentrate compared with placebo in acute hereditary angioedema attacks. J Allergy Clin Immunol 2009; 124:801-808.

-9 Zuraw B, Cicardi M, Levy RJ, et al: Recombinant human C1INH for the treatment of acute angioedema attacks in patients with hereditary angioedema. J Allergy Clin Immunol 2010;126:821-827, e14.

-10 Waytes AT, Rosen FS, Frank MM: Treatment of hereditary angioedema with a vapor-heated C1INH concentrate. N Engl J Med 1996; 334:1630-1634.

-11 Kunschak M, Engl W, Maritsch F, et al: A randomized, controlled trial to study the efficacy and safety of C1INH concentrate in treating hereditary angioedema. Transfusion 1998;38:540-549.
12 Bork K, Barnstedt SE: Treatment of 193 episodes of laryngeal edema with C1INH concentrate in patients with hereditary angioedema. Arch Intern Med 2001;161:714-718.

13 Cicardi M, Zingale L: How do we treat patients with hereditary angioedema. Transfus Apher Sci 2003;29:221-227.

14 Farkas H, Jakab L, Temesszentandrasi G, et al: Hereditary angioedema: a decade of human C1INH concentrate therapy. J Allergy Clin Immunol 2007;120:941-947.

15 Bork K, Meng G, Staubach P, Hardt J: Treatment with C1INH concentrate in abdominal pain attacks of patients with hereditary angioedema. Transfusion 2005;45:1774-1784.

16 Bright P, Gompels M, Demptster J, Longhurst H: Case series: UK experience of icatibant for acute attacks of hereditary angioedema. Norwich, EAACI summer school, 2009.

17 Kreuz W, Martinez-Saguer I, Aygoren-Pursun E, Rusicke E, Heller C, Klingebiel T: $\mathrm{C} 1 \mathrm{INH}$ concentrate for individual replacement therapy in patients with severe hereditary angioedema refractory to danazol prophylaxis. Transfusion 2009;49:1987-1995.

18 Bygum A, Andersen KE, Mikkelsen CS: Selfadministration of intravenous $\mathrm{C} 1 \mathrm{INH}$ therapy for hereditary angioedema and associated quality of life benefits. Eur J Dermatol 2009;19:147-151.

19 Levi M, Choi G, Picavet C, Hack CE: Selfadministration of C1INH concentrate in patients with hereditary or acquired angioedema caused by C1INH deficiency. J Allergy Clin Immunol 2006;117:904-908.

20 Longhurst HJ, Carr S, Khair K: C1INH concentrate home therapy for hereditary angioedema: a viable, effective treatment option. Clin Exp Immunol 2007;147:11-17.
21 Agostoni A, Aygoren-Pursun E, Binkley KE, et al: Hereditary and acquired angioedema: problems and progress: proceedings of the third C1 esterase inhibitor deficiency workshop and beyond. J Allergy Clin Immunol 2004;114:S51-S131.

22 Kreuz W, Martinez-Saguer I, Rusicke E, Aygoren-Pursun E, Klingebiel T: Impact of the Frankfurt HAE therapy protocol on healthrelaed quality of life (HRQoL) in 50 patients with hereditary angioedema. Abstract 6th Eurpean C1INH Deficiency Workshop, Budapest, 2009.

23 Zuraw B, Davis D, Castaldo A: Safety and efficacy of physician supervised self-managed $\mathrm{C} 1 \mathrm{INH}$ individual replacement therapy. Abstract 6th European C1INH Deficiency Workshop, Budapest, 2009.

24 Bork K, Staubach P, Eckardt AJ, Hardt J: Symptoms, course, and complications of abdominal attacks in hereditary angioedema due to C1INH deficiency. Am J Gastroenterol 2006;101:619-627.

25 Longhurst HJ, Farkas H, Craig T, et al: HAE international home therapy consensus document. Allergy Asthma Clin Immunol 2010;6: 22.

26 Teitel JM, Barnard D, Israels S, Lillicrap D, Poon MC, Sek J: Home management of haemophilia. Haemophilia 2004;10:118-133.

$\checkmark 27$ Caballero T, Lopez-Serrano C: Anaphylactic reaction and antibodies to DX-88 (kallikrein inhibitor) in a patient with hereditary angioedema. J Allergy Clin Immunol 2006;117: 476-477, discussion 7.

28 Ad Hoc Committee on Medical Ethics: American College of Physicians Ethics Manual. Part 1: history of medical ethics, the physician and the patient, the physician's relationship to other physicians, the physician and society. Ann Intern Med 1984;101:129137.

-29 Waterwork SLK: Reluctant collaborators: do patients want to be involved in decision concerning care? J Adv Nurse 1990;15:971-976. 